\title{
XLV. Note on some thermal properties of a mixture of carbonic acid and nitrogen
}

\section{K. Tsuruta}

To cite this article: K. Tsuruta (1893) XLV. Note on some thermal properties of a mixture of carbonic acid and nitrogen, Philosophical Magazine Series 5, 36:222, 438-453, DOI: $10.1080 / 14786449308620500$

To link to this article: http://dx.doi.org/10.1080/14786449308620500

曲 Published online: 08 May 2009.

Submit your article to this journal $[\pi$

Џ Article views: 3

Q View related articles $\asymp$ 


\section{[ 438 ]}

XLV. Note on some Thermal Properties of a Mixture of Carbonic Acid and Nitrogen. By K.TsuruTA, Rigakushi, Tokio, Japan*.

\section{Literature.}

IN $\mathrm{N}$ his classical researches on thermal properties of carbonic acid, Andrews found the isothermals for $13^{\circ} \cdot 1$ and $21^{\circ} \cdot 5$ a little rounded-off at the points of complete liquefaction and of complete evaporation, and pointed out that this was due to a very small unavoidable admixture of air in the gas. Maxwell was much interested in this "question, of an exceedingly interesting nature," as appears from his letters to Andrews $†$. Andrews then made a further investigation on the subject, the results of which were given in an abstract $\neq$. A further account was published as a posthumous paper in the 'Philosophical Transactions' for $1886 \S$.

The subject did not remain unattacked by other physicists working in this part of the field of investigation. Cuilletet 7 made some experiments as early as 1880 on mixtures of carbonic acid and other gases, on which Jamin wrote a note ${ }^{* *}$. In England, Ansdell was occupied with various mixtures of hydrochloric acid and carbonic acid $\dagger \dagger$.

Cailletet studied especially the very curions fact of the appearance and disappearance of the condensed state at certain temperatures and pressures; Ansdell confined his attention to the determinations of the critical temperatures of the mixtures ; while Dewar described many interesting facts relating to the behaviour of carbonic acid in presence of other substances $\ddagger$. Andrews's paper 'contains a series of measurements on changes of volumes, pressures, and temperatures of the mixture of carbonic acid and nitrogen in the proportion of 3 to 4 . Thus, although the paper was published, according to Prof. Tait, twelve years after the date of experiment, yet it has remained, and still remains, so far as I am aware, a very complete investigation and perhaps the only one.

* Communicated by the Author.

+ The Memoir on the Life of T. Andrews, by Tait and Brown, pp. 14-15.

$\ddagger$ 'The Scientific Papers of T. Andrews,' pp. 383-392.

Ibid. pp. 457-471.

IT Journ. de Phys. tom. ix. (1880).

* Comptes Rendus, xcvi. (1883).

t† Proc. Roy. Soc. vol. xxxiv. (1882).

$\ddagger$ Ibid. vol. xxx. (1880). 
Soon after the publication of the posthumous paper, Herr Margules* calculated some thermodynamical magnitudes based on the data supplied. Herr Galitzene $\dagger$ developed the incomplete result of comparison made by Andrews himself between the experimental data and Dalton's law of gaseous mixtures.

\section{Characteristic Equation.}

We are not at present in possession of a rational form of characteristic equation applicable to all substances. Some of those which have been proposed as such hold good only for one class of substances, nor do they continue to hold strictly through the whole range of physical changes within the reach of experiment even in the case of the very substances for which they were particularly given. They are therefore to be looked upon as empirical and tentative, and we have to make use of them as such for the time being, or to regard them as interpolation formulæ whose mathematical forms are much like those of a rational one. In choosing the Clausius' form of characteristic equation, I was guided by no other consideration than the above. It is

$$
p=\frac{\mathrm{RT}}{v-\alpha}-\frac{\Theta}{(v+\beta)^{2}}
$$

in which $\Theta$ is a certain undetermined function of $\mathrm{T}$, the simplest form being

$$
\Theta=\frac{K(a \text { constant })}{T} \text {. }
$$

In the first place I have to determine the four constants $\mathrm{R}, \mathrm{K}, \alpha$, and $\beta$ for the mixture under consideration.

As Andrews used a hydrogen-manometer, and hydrogen presents a decided deviation from the gaseous laws, corrections for the given manometric values of pressure are to be applied. It is certain, although not explicitly mentioned, that Andrews calculated pressures by the equation $\ddagger$

$$
p=\frac{V_{0}(1+\alpha t)}{V_{1}} \mp \frac{q}{760},
$$

in which $\nabla_{0}$ is the volume of the mixture at $0^{\circ}$ and under the normal pressure, $V_{1}$ that at the temperature $t$ and under the

* Wiener Sitzangsbericht, Bd. xcvii. (1888).

$\dagger$ Wied. Ann. Bd. xci. (1890).

$\ddagger$ 'l'he Scientific Papers,' p. 422. 
pressure $p$, and $q$ denotes the difference of mercury-levels in the manometer and the tube containing the mixture. $V_{1}$ was not given by Andrews, so that I must proceed in the inverse manner to deduce $\mathrm{V}_{1}$ from $p$ according to the above formula. $q / 760$ is an unknown factor; but inasmuch as Andrews mentions in his first Bakerian Lecture that the difference rarely exceeded $200 \mathrm{~mm}$., a correction arising from this is so small that it can be safely neglected. For example, since

$$
\frac{\mathrm{V}_{1}}{\overline{\mathrm{V}}_{0}}=\frac{1}{p}+\frac{\alpha t}{p} \mp \frac{q}{760 \times p^{2}}
$$

taking the greatest possible value, $200 \mathrm{~mm}$., for $q$, and the least possible value, 40 atmospheres, for $p$, we have

$$
\frac{q}{760 \times p^{2}}=0.000164 \text {. }
$$

Thus I get the values of $V_{1}$, which will not perhaps much differ from the directly observed ones.

We are now able to calculate the corrections for the manometric readings by applying the Clausius' form of characteristic equation for hydrogen with the values of the constants determined by Sarrau "based on the data supplied by Amagat's classical research-

$$
\begin{gathered}
\mathrm{R}=0.003665, \quad \mathrm{~K}=0.0551, \quad \alpha=0.000889, \\
\beta=-0.00043 .
\end{gathered}
$$

I have myself found that these values are very good, not only in the few instances given by Sarrau, but also throughout.

Although I was satisfied so far, yet I was anxious to extend the verification to the temperatures of our manometer, $7^{\circ} \cdot 2$, $7^{\circ} .4,12^{\circ} \cdot 0,12^{\circ} .8$, the temperature-range in Amagat's investigation being from $30^{\circ}$ to $100^{\circ}$. Wroblewski's posthumous paper $t$ on the compressibility of hydrogen was the only one available for me. I give below so much as is thought sufficient of my results of comparison:-

* Comptes Rendus, xciv. (1882).

† Wiener Sitzungsbericht, Bd. xcvii. (1888). 
a Mirture of Carbonic Acid and Nitrogen.

\begin{tabular}{|c|c|c|c|}
\hline$t$ & $\begin{array}{l}\text { Wroblewski's } \\
\text { measurements. }\end{array}$ & $\begin{array}{c}\text { Clausius, Amagat, } \\
\text { and Sarrau. }\end{array}$ & Diff. \\
\hline $\begin{array}{l}8 \\
" \\
" \\
"\end{array}$ & $\begin{array}{c}\text { atm. } \\
29 \cdot 635 \\
39 \cdot 355 \\
49 \cdot 097 \\
68 \cdot 791\end{array}$ & $\begin{array}{l}\text { atm. } \\
29 \cdot 488 \\
38 \cdot 654 \\
48 \cdot 050 \\
67 \cdot 641\end{array}$ & $\begin{array}{l}-0 \cdot 147 \\
-0 \cdot 701 \\
-1 \cdot 047 \\
-1 \cdot 150\end{array}$ \\
\hline $\begin{array}{c}99 \cdot 14 \\
" \\
" \\
"\end{array}$ & $\begin{array}{r}1.000 \\
19.552 \\
49.223 \\
68.582\end{array}$ & $\begin{array}{r}1 \cdot 006 \\
19 \cdot 516 \\
48 \cdot 565 \\
67 \cdot 702\end{array}$ & $\begin{array}{r}+0.006 \\
-0.036 \\
-0.658 \\
-0.880\end{array}$ \\
\hline
\end{tabular}

The method of Wroblewski was quite different from that of Amagat, yet their results agree sufficiently well with one another.

I then proceeded to the calculation of pressures by equation (1). The results are contained in the following Tables; the corrected values in I., II., III. agree very well with those given by Margules and Galitzene, but those in IV. show some, though small differences.

TABLE I.

\begin{tabular}{|c|c|c|c|}
\hline$t$. & $\nabla_{1}$. & $\underset{\text { given. }}{P}$ & $\begin{array}{c}\mathrm{P}, \\
\text { corrected. }\end{array}$ \\
\hline 7.30 & 0.024416 & $42 \cdot 05$ & $43 \cdot 30$ \\
\hline $7 \cdot 30$ & 0.021091 & 48.68 & 50.36 \\
\hline $7 \cdot 22$ & 0.019877 & $51 \cdot 64$ & 53.54 \\
\hline $7 \cdot 26$ & 0.018755 & $54 \cdot 73$ & $56 \cdot 87$ \\
\hline $7 \cdot 21$ & 0.017566 & 58.43 & $60 \cdot 88$ \\
\hline $7 \cdot 21$ & 0.016388 & $62 \cdot 63$ & $65 \cdot 45$ \\
\hline $7 \cdot 20$ & 0.015272 & $67 \cdot 20$ & 70.47 \\
\hline $7 \cdot 18$ & 0.013247 & $79 \cdot 47$ & $81 \cdot 66$ \\
\hline $7 \cdot 17$ & 0.011698 & 87.73 & $93 \cdot 40$ \\
\hline $7 \cdot 17$ & 0.010557 & $97 \cdot 21$ & $104 \cdot 23$ \\
\hline $7 \cdot 17$ & 0.009450 & $108 \cdot 60$ & $117 \cdot 46$ \\
\hline $7 \cdot 17$ & 0.008316 & $123 \cdot 40$ & 135.01 \\
\hline
\end{tabular}


TABLE II.

\begin{tabular}{|c|c|c|c|}
\hline$t$ & $\mathbf{V}_{\mathrm{i}}$ & $\underset{\text { given. }}{\mathbf{P}}$ & $\stackrel{P}{\text { corrected. }}$ \\
\hline $\begin{array}{l}7 \cdot 47 \\
7 \cdot 48 \\
7 \cdot 42 \\
7 \cdot 46 \\
7 \cdot 48 \\
7 \cdot 42 \\
7 \cdot 54 \\
7 \cdot 50 \\
7 \cdot 48 \\
7 \cdot 59 \\
7 \cdot 65 \\
7 \cdot 55 \\
7 \cdot 80 \\
7 \cdot 54 \\
7 \cdot 58 \\
7 \cdot 79 \\
7 \cdot 63 \\
7 \cdot 67 \\
7 \cdot 58\end{array}$ & $\begin{array}{l}0.025890 \\
0.024714 \\
0.023526 \\
0.022332 \\
0.019957 \\
0.018761 \\
0 \cdot 017644 \\
0.016450 \\
0.015320 \\
0.014079 \\
0.012976 \\
0.011785 \\
0.010660 \\
0.009454 \\
0.007134 \\
0.006181 \\
0.005928 \\
0.004766 \\
0.003620\end{array}$ & $\begin{array}{r}39 \cdot 68 \\
41 \cdot 56 \\
43 \cdot 66 \\
46 \cdot 00 \\
\mathbf{0} 1 \cdot 48 \\
54 \cdot 75 \\
58 \cdot 24 \\
62 \cdot 46 \\
67 \cdot 07 \\
73 \cdot 00 \\
79 \cdot 22 \\
87 \cdot 20 \\
96 \cdot 50 \\
108 \cdot 70 \\
144 \cdot 00 \\
166 \cdot 40 \\
173 \cdot 40 \\
215 \cdot 70 \\
28390\end{array}$ & $\begin{array}{r}40 \cdot 79 \\
42 \cdot 79 \\
44 \cdot 14 \\
47 \cdot 61 \\
53 \cdot 36 \\
56 \cdot 89 \\
60 \cdot 67 \\
65 \cdot 27 \\
70 \cdot 31 \\
76 \cdot 87 \\
83 \cdot 80 \\
92 \cdot 80 \\
103 \cdot 43 \\
117 \cdot 54 \\
160 \cdot 20 \\
188 \cdot 42 \\
197 \cdot 50 \\
254 \cdot 73 \\
357 \cdot 17\end{array}$ \\
\hline
\end{tabular}

TABLE III.

\begin{tabular}{|c|c|c|c|}
\hline$t$ & $\nabla_{1}$ & $\underset{\text { given. }}{\mathbf{P}}$ & $\stackrel{P,}{\text { corrected. }}$ \\
\hline $11 \cdot 61$ & 0.024668 & $42 \cdot 26$ & $43 \cdot 51$ \\
\hline 11.97 & 0021115 & $49 \cdot 43$ & $51 \cdot 15$ \\
\hline $11 \cdot 86$ & $0 \cdot 018692$ & $55 \cdot 82$ & $58 \cdot 03$ \\
\hline $12 \cdot 38$ & 0.016366 & $63 \cdot 87$ & $66 \cdot 78$ \\
\hline $12 \cdot 38$ & 0.014450 & $72 \cdot 34$ & $76 \cdot 12$ \\
\hline $12 \cdot 38$ & 0.011711 & $89 \cdot 26$ & $95 \cdot 08$ \\
\hline $11 \cdot 96$ & 0009489 & 11000 & $119 \cdot 02$ \\
\hline $11 \cdot 63$ & 0.007160 & $145 \cdot 60$ & $161 \cdot 76$ \\
\hline 1170 & 0004704 & $221 \cdot 70$ & 26278 \\
\hline
\end{tabular}

TABLe IV.

\begin{tabular}{|c|c|c|c|}
\hline$t$ & $\nabla_{1}$. & $\begin{array}{c}P \\
\text { given. }\end{array}$ & $\underset{\text { corrected. }}{\mathrm{P}}$ \\
\hline$\stackrel{\circ}{8} 39$ & 0.024600 & $47 \cdot 90$ & $43 \cdot 14$ \\
\hline $8 \cdot 42$ & 0.021123 & $48 \cdot 80$ & $50 \cdot 49$ \\
\hline 1206 & $0 \cdot 018692$ & $55 \cdot 86$ & 58.07 \\
\hline $12 \cdot 08$ & $0 \cdot 016270$ & $64 \cdot 18$ & $67: 13$ \\
\hline 1218 & $0 \cdot 014400$ & 72.54 & $76 \cdot 33$ \\
\hline $12 \cdot 36$ & 0.009459 & $110 \cdot 50$ & $119 \cdot 60$ \\
\hline $12 \cdot 3 t$ & 0007105 & $147 \cdot 10$ & 16381 \\
\hline $12-40$ & 0.004675 & $223 \cdot 60$ & $265 \cdot 41$ \\
\hline
\end{tabular}


The constants $\mathrm{R}, \mathrm{K}, \alpha, \beta$ were calculated by the method of least squares, taking into account all the measurements given by Andrews, altogether 48 in number. Their values actually found by me were a little different from those given by Margules, which latter are :-

$$
\begin{array}{ll}
\mathrm{R}=0.003674, & \mathrm{~K}=0.953, \\
\alpha=0.0014, & \beta=0.00015 .
\end{array}
$$

But when I compared the values of pressure calculated with my values of the constants with the above corrected values, I found that Margules' values are on the whole rather preferable to mine, so that, to avoid useless diversities, I determined to adopt Margules' values throughout the following pages.

The following Table contains, besides the corrected results of Andrews's measurements, the calculated values of pressure according to equation (1) with the above values of the constants; it contains all data that are wanted to get isothermals, jsomerics, and isopiestics of the mixture. It will be seen how well equation (1) represents the results of measurements, and so all that is possibly contained in it may as reasonably be accepted as the measurements themselves.

\begin{tabular}{|c|c|c|c|c|}
\hline$t$. & $v$. & $p$ (obs.). & $p$ (calc.) & Diff. \\
\hline $2 \cdot 32$ & 0.02138 & $\begin{array}{l}\text { atm. } \\
43 \cdot 30\end{array}$ & $\begin{array}{l}\text { atm. } \\
43 \cdot 16\end{array}$ & -0.14 \\
\hline 234 & 0.01805 & $50 \cdot 36$ & $50 \cdot 31$ & -0.05 \\
\hline $2 \cdot 08$ & 0.01681 & 53.54 & 53.54 & 0.00 \\
\hline $2 \cdot 38$ & 0.01575 & $56 \cdot 87$ & $56 \cdot 82$ & -0.05 \\
\hline $2 \cdot 06$ & 0.01455 & 6088 & $60 \cdot 83$ & -0.05 \\
\hline $2 \cdot 10$ & 0.01338 & $65 \cdot 45$ & 65.44 & -001 \\
\hline $2 \cdot 16$ & 0.01229 & $70 \cdot 47$ & 7045 & -0.02 \\
\hline $2 \cdot 21$ & 0.00998 & $84 \cdot 08$ & $8+\cdot 10$ & +0.02 \\
\hline $2 \cdot 21$ & 000881 & $93 \cdot 40$ & $93 \cdot 32$ & -0.08 \\
\hline $2 \cdot 17$ & 0.00772 & $104 \cdot 23$ & $104 \cdot 05$ & -0.18 \\
\hline $2 \cdot 21$ & 0.00668 & $117 \cdot 46$ & $117 \cdot 27$ & -0.19 \\
\hline $2 \cdot 25$ & 0.00568 & 135.01 & $134 \cdot 41$ & -0.60 \\
\hline
\end{tabular}

\section{Table I.}


TABLE II.

\begin{tabular}{|c|c|c|c|c|}
\hline$t$. & $v$. & $p($ obs. $)$ & $p$ (calc) & Diff. \\
\hline $7 \cdot 50$ & 0.02350 & $\begin{array}{l}\text { atm. } \\
4079\end{array}$ & atm. & -0.23 \\
\hline 7.50 & 0.02231 & $42 \cdot 79$ & $42 \cdot 55$ & -0.24 \\
\hline $7 \cdot 50$ & 0.02109 & 4501 & $44 \cdot 81$ & $-0 \cdot 20$ \\
\hline 7.53 & 0.01987 & $47 \cdot 61$ & 47.34 & $-0 \cdot 27$ \\
\hline 7.51 & 001744 & $53 \cdot 36$ & $53 \cdot 27$ & -0.09 \\
\hline $7 \cdot 59$ & 0.01625 & 56.89 & 56.78 & -0.11 \\
\hline $7 \cdot 50$ & 0.01512 & 6067 & $60 \cdot 54$ & -0.13 \\
\hline 7.50 & 001391 & $65 \cdot 27$ & $65 \cdot 19$ & -0.08 \\
\hline $7 \cdot 50$ & 0.01274 & 7031 & $70 \cdot 43$ & +0.12 \\
\hline 7.50 & 0.01149 & 76.87 & 77.06 & +0.19 \\
\hline $7 \cdot 51$ & 001054 & $83 \cdot 80$ & 83.03 & -077 \\
\hline 7.50 & 0.00926 & $92 \cdot 80$ & $92 \cdot 75$ & -005 \\
\hline $7 \cdot 08$ & 0.00808 & $103 \cdot 43$ & 103.81 & +0.38 \\
\hline 748 & 0.00691 & $117 \cdot 54$ & $118 \cdot 15$ & $+0 \cdot 61$ \\
\hline $7 \cdot 54$ & 0.00495 & 160.20 & $159 \cdot 74$ & -0.46 \\
\hline 7.50 & 000422 & 188.42 & 187.54 & -0.88 \\
\hline $7 \cdot 49$ & 0.00404 & $197 \cdot 50$ & $196 \cdot 82$ & -068 \\
\hline $7 \cdot 50$ & 0.00332 & $254 \cdot 73$ & $254 \cdot 58$ & -0.15 \\
\hline & 0.00272 & $357 \cdot 17$ & $368 \cdot 21$ & +11.04 \\
\hline
\end{tabular}

Table III.

\begin{tabular}{|c|c|c|c|c|}
\hline$t$. & $v$. & $p$ (obs.). & $p$ (calc.) & Diff. \\
\hline $3 i \cdot 35$ & 0.02445 & atm. & atm. & $-0 \cdot 17$ \\
\hline 31.31 & 0.02055 & $51 \cdot 15$ & 51.07 & -0.08 \\
\hline $31 \cdot 21$ & 0.01795 & $58 \cdot 03$ & 57.97 & -0.06 \\
\hline $31 \cdot 40$ & 0.01542 & 6678 & $66 \cdot 85$ & +0.07 \\
\hline $31 \cdot 14$ & 0.01337 & $76 \cdot 12$ & 76.21 & +0.09 \\
\hline 31.06 & 0.01045 & 95.08 & 95.57 & +0.49 \\
\hline $31 \cdot 36$ & 0.00816 & $119 \cdot 02$ & 120.07 & +1.05 \\
\hline $31 \cdot 35$ & 0.00586 & $161 \cdot 76$ & 16403 & $+2 \cdot 27$ \\
\hline $31 \cdot 30$ & 0.00377 & 262.78 & 267.92 & $+5 \cdot 14$ \\
\hline
\end{tabular}

TABLE IV.

\begin{tabular}{|c|c|c|c|c|}
\hline$t$. & $v$. & $p$ (obs.). & $p$ (calc.). & Diff. \\
\hline 19.2\% & 0.09035 & atm. & atm. & \\
\hline $48 \cdot 11$ & 002239 & 50.49 & 5036 & -0.13 \\
\hline $48 \cdot 48$ & 0.01929 & 58.07 & $58 \cdot 18$ & +0.11 \\
\hline 48.43 & 0.01662 & $67 \cdot 13$ & 67.05 & -0.08 \\
\hline 48.66 & 0.01448 & $76 \cdot 33$ & 76.51 & +0.18 \\
\hline $48 \cdot 38$ & 000896 & 11960 & $120 \cdot 45$ & +0.85 \\
\hline 48.49 & 0.00646 & 16381 & 165.58 & +1.77 \\
\hline $48 \cdot 47$ & 0.00430 & $265 \cdot 41$ & $257 \cdot 57$ & -7884 \\
\hline
\end{tabular}


Isotuermals, Isometrics, and Isopiestics of the Mixture.

Having obtained the characteristic equation of the mixture, 1 proceeded to construct a series of isothermals, the parametric values $\left(2^{\circ} \cdot 2,7^{\circ} \cdot 5,31^{\circ} \cdot 3,48^{\circ} \cdot 4\right)$ of four of which were chosen according to Andrews.

Table I. $2^{\circ} \cdot 2$.

\begin{tabular}{|c|c|c|c|c|c|}
\hline$p$. & $v$. & $p v$. & $p$. & $v$. & $p v$. \\
\hline 43.13 & 0.02138 & 0.92212 & 70.30 & 001229 & 0.86399 \\
50.27 & 0.01805 & 0.90737 & 83.82 & 0.00998 & 0.83652 \\
53.57 & 0.01681 & 0.90101 & 93.31 & 0.00881 & 0.82206 \\
56.63 & 001575 & 0.89192 & 104.07 & 0.00772 & 0.80342 \\
60.86 & 0.01455 & 0.88551 & 117.26 & 0.00668 & $0 \cdot 78330$ \\
65.48 & 0.01338 & 0.87612 & 134.35 & 0.00568 & 0.76311 \\
\hline
\end{tabular}

TABLE 1I. $7^{\circ} \cdot 5$.

\begin{tabular}{|c|c|c|c|c|c|}
\hline$p$ & $v$ & $p v$. & $p$. & $v$. & $p v$. \\
\hline 40.56 & 0.02350 & 0.95316 & 118.87 & $0 \cdot 00691$ & 0.82139 \\
\hline 42.55 & 0.02231 & 0.94929 & $127 \cdot 61$ & 0.00636 & $0.81160 *$ \\
\hline 44.81 & 002109 & 0.94504 & $143 \cdot 52$ & 0.00556 & 0.79797 * \\
\hline $47 \cdot 32$ & 0.01987 & 0.94025 & 15223 & 0.00521 & 0.79312 * \\
\hline $53 \cdot 27$ & 0.01744 & 0.92903 & $159 \cdot 67$ & 0.00495 & 079037 \\
\hline 56.77 & 001625 & 0.92251 & $176^{\circ} 92$ & 0.00446 & 0.78906 * \\
\hline $60 \cdot 54$ & 0.01512 & 0.91536 & 187.54 & 0.00422 & 0.79142 \\
\hline $65 \cdot 19$ & 0.01391 & 0.90679 & 196.84 & 0.00404 & 0.79523 \\
\hline $70 \cdot 43$ & 0.01274 & 088728 & $220 \cdot 39$ & 0.00368 & $0-81108$ * \\
\hline 77.06 & 0.01149 & 0.88542 & 254.58 & 000332 & 0.84520 \\
\hline 83.02 & 001054 & 0.87503 & $281 \cdot 43$ & 0.00312 & 0.87806 \\
\hline 92.74 & 0.00926 & 0.85877 & 324.02 & 0.00289 & 0.93642 \\
\hline $104 \cdot 11$ & 0.00808 & 0.84121 & $368 \cdot 25$ & 0.00272 & 0.00164 \\
\hline
\end{tabular}

TABLE III. $31^{\circ} \cdot 3$.

\begin{tabular}{|c|c|c|}
\hline$p$. & \multicolumn{1}{c|}{$v$} & \multicolumn{1}{c|}{$p v}$. \\
\hline 43.33 & 0.02445 & 1.05942 \\
51.07 & 0.02055 & 1.04949 \\
57.99 & 0.01795 & 1.04092 \\
66.82 & 0.01542 & 1.03036 \\
76.27 & 0.01337 & 1.01973 \\
$95 \cdot 66$ & 0.01045 & 0.99965 \\
120.03 & 0.00816 & 0.97944 \\
138.20 & 0.00701 & $0.96878 *$ \\
163.97 & 0.00586 & 0.96086 \\
179.17 & 0.00536 & $0.9603 \% *$ \\
$205 \cdot 17$ & 0.00471 & $0.96635 *$ \\
223.73 & 0.00436 & $0.97546 *$ \\
244.34 & 0.00405 & $098958 *$ \\
267.92 & 000377 & 1.01006 \\
\hline
\end{tabular}

TABLE IV. $48^{\circ} \cdot 4$.

\begin{tabular}{|c|c|c|}
\hline$p$ & $v$ & $p v$ \\
\hline $43 \cdot 10$ & 0.02635 & $1 \cdot 13568$ \\
\hline $50 \cdot 42$ & 0.02239 & $1 \cdot 12890$ \\
\hline $5 K \cdot 16$ & $0 \cdot 01929$ & $1 \cdot 12190$ \\
\hline 67.04 & 001662 & $1 \cdot 11420$ \\
\hline $76 \cdot 42$ & 0.01448 & $1 \cdot 10656$ \\
\hline 92.93 & 0.01178 & $1.09471 *$ \\
\hline $120 \cdot 46$ & 0.00896 & $1 \cdot 07932$ \\
\hline $138 \cdot 28$ & 0.00776 & $1 \cdot 07305 *$ \\
\hline 165.50 & 0.00646 & ] 06913 \\
\hline $169 \cdot 71$ & 0.00630 & $1 \cdot 06917 *$ \\
\hline $178 \cdot 30$ & 0.00600 & $1.06930_{*}$ \\
\hline $189 \cdot 70$ & 0.00565 & 1.07180 \\
\hline $226 \cdot 29$ & 0.00480 & $1.08619 *$ \\
\hline $257 \cdot 44$ & 0.00436 & $1 \cdot 10699$ \\
\hline
\end{tabular}


The isothermals in fig. 1 are plotted from these numbers, of which those distinguished by stars are simply supplied from the characteristic equation. They are considerably removed from the critical temperature of the mixture, which was

Fig. 1.

The Isothermals.

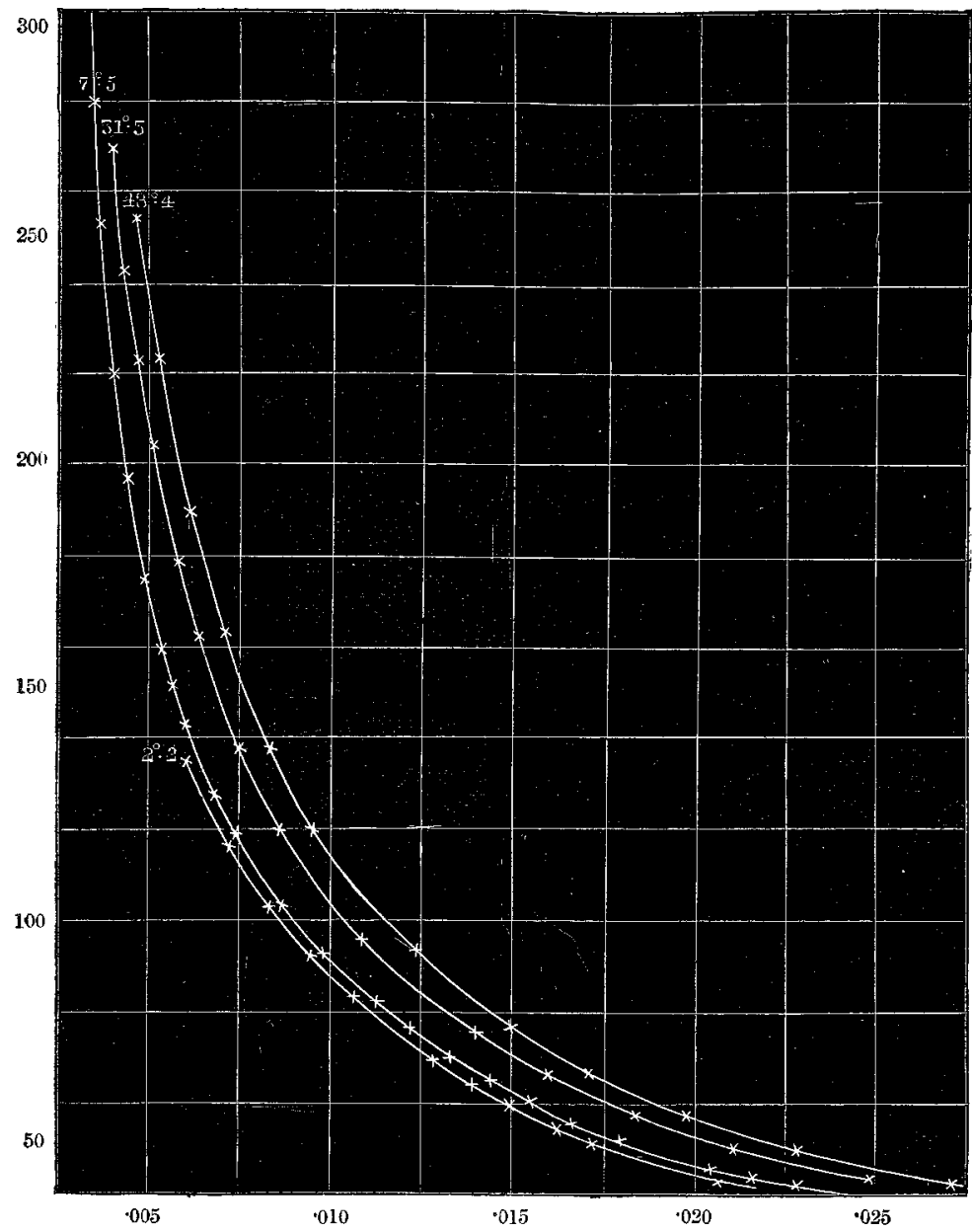

found by Andrews to be below $-20^{\circ}$. Inasmuch as they are so regular over a wide range of temperature, we shall not go far wrong in extending our results of measurements within a few degrees of temperature above and below that range. 
M. Amagat makes use of isothermals of another type, of which the product $p v$ is taken for ordinate, and the pressure $p$ for abscissa. For the sake of comparison they are drawn in fig. 2. The product $p v$ has a minimum value for each

Fig. 2.

$p$ and $p v$ lines.

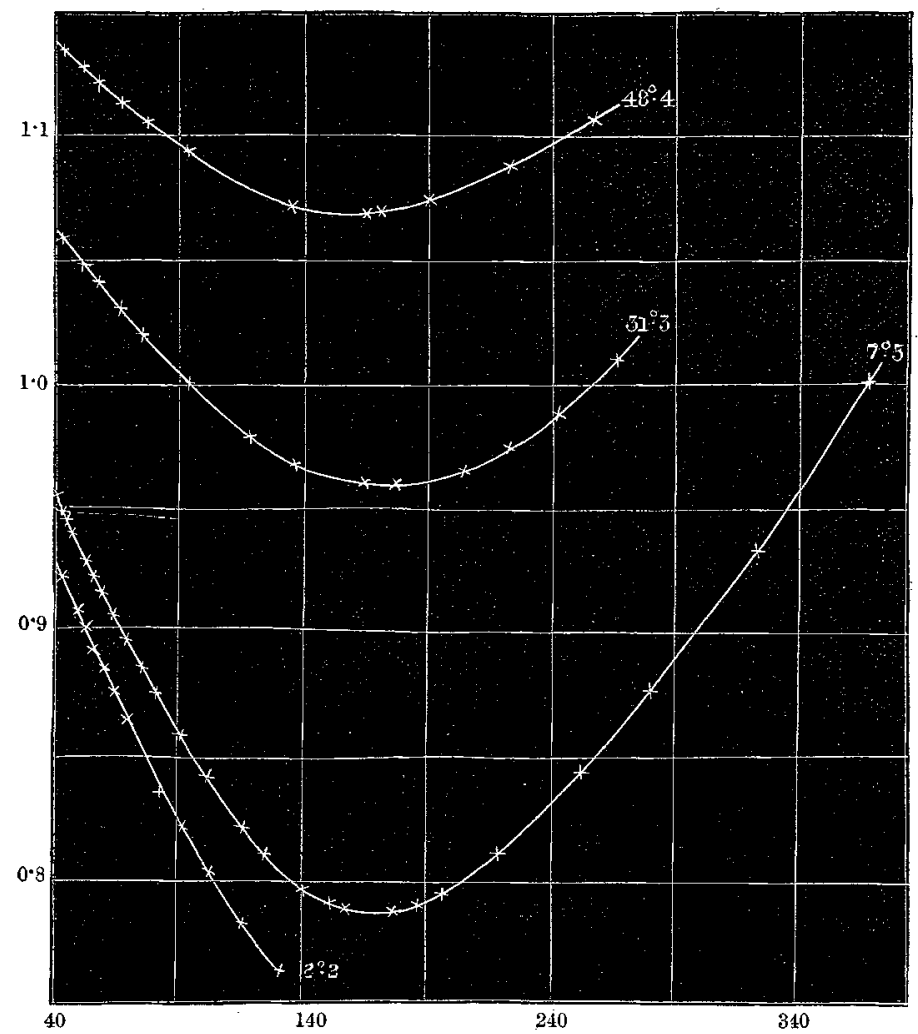

temperature. The parts of the isothermals which are on the left-hand side of that value are appreciably convex towards the origin, while those which are on the right-hand side seem to converge asymptotically, their curvatures tending to become zero. These features belong to isothermals of this type for pure carbonic acid at much higher temperatures ; thus an admixture of a more permanent gas like nitrogen seems to have the same effect as a rise of temperature, as a priori would be probable. The locus of these minima can be found from the characteristic equation, but its equation is almost uselessly complex. The following values were found by trials :- 


\begin{tabular}{|c|c|c|c|}
\hline$t$. & $p$. & $v$. & $p v$. \\
\hline 7.5 & 171.65 & 0.004595 & 0.78873 \\
31.3 & 174.07 & 0.005516 & 0.96017 \\
48.4 & 166.40 & 0.006425 & 1.06912 \\
\hline
\end{tabular}

Thus the locus is convex towards the axis of $p v$, and there is a mini-max point; in fact the existence of such a point was already pointed out by M. Amagat, for the case of pure carbonic acid.

Fig. 3.

Isometrics.

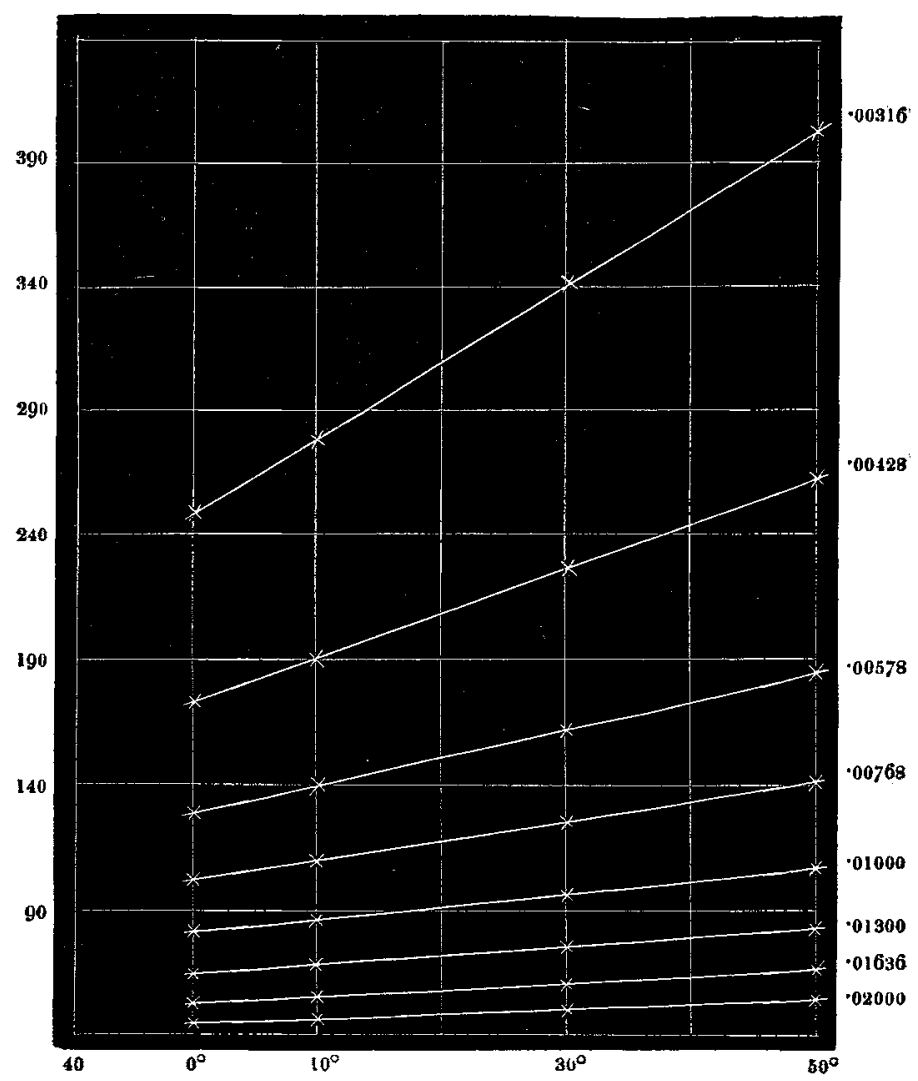

The parametric values of volumes and pressures for isometrics and isopiestics respectively are so chosen that we can easily compare the results with those for pure carbonic acid, as given by the most recent experiments of $M$. Amagat* (see fig. 3 ).

* Comptes Rendus, 1891. 


\begin{tabular}{|c|c|c|c|c|c|c|c|c|}
\hline \multicolumn{2}{|c|}{$0^{\circ}$. } & \multicolumn{2}{|c|}{$10^{\circ}$} & \multicolumn{2}{|c|}{$30^{\circ}$. } & \multicolumn{2}{|c|}{$50^{\circ}$} & \multirow{2}{*}{$v$} \\
\hline Mixture. & $\begin{array}{l}\text { Pure } \\
\text { Gas. }\end{array}$ & Mixture & $\begin{array}{l}\text { Pure } \\
\text { Gas. }\end{array}$ & Mixture. & $\begin{array}{l}\text { Pure } \\
\text { Gas. }\end{array}$ & Mixture. & $\begin{array}{l}\text { Pure } \\
\text { Gas. }\end{array}$ & \\
\hline $\begin{array}{l}\text { atm. } \\
45 \cdot 33\end{array}$ & $\begin{array}{l}\text { atm. } \\
\text {....... }\end{array}$ & $\begin{array}{l}\text { atm. } \\
47 \cdot 60\end{array}$ & $\begin{array}{l}\text { atm. } \\
\ldots \ldots . .\end{array}$ & $\begin{array}{l}\text { atm. } \\
52 \cdot 10\end{array}$ & $\begin{array}{l}\text { atm. } \\
\text {....... }\end{array}$ & $\begin{array}{l}\text { atm. } \\
56.53\end{array}$ & $\begin{array}{l}\text { atm. } \\
\text {....... }\end{array}$ & 0.02000 \\
\hline $54 \cdot 24$ & $34 \cdot 4$ & $57 \cdot 15$ & $41 \cdot 8$ & $62 \cdot 87$ & $48 \cdot 3$ & $68 \cdot 50$ & $54 \cdot 5$ & 0.01636 \\
\hline $66 \cdot 28$ & $34 \cdot 4$ & $70 \cdot 16$ & $44 \cdot 4$ & $77 \cdot 78$ & $55 \cdot 5$ & $85 \cdot 24$ & $63 \cdot 8$ & 0.01300 \\
\hline $82 \cdot 74$ & $34 \cdot 4$ & $88 \cdot 21$ & $44 \cdot 4$ & 98.91 & $62 \cdot 8$ & $100 \cdot 35$ & 745 & $0 \cdot 01000$ \\
\hline $102 \cdot 78$ & $34 \cdot 4$ & 11066 & $44 \cdot 4$ & 125.96 & $68 \cdot 3$ & $140 \cdot 84$ & $84 \cdot 8$ & 0.00768 \\
\hline 12973 & $34 \cdot 4$ & $141 \cdot 62$ & $44 \cdot 4$ & 16472 & 707 & $187 \cdot 03$ & 9477 & 0.00578 \\
\hline $170 \cdot 39$ & $34 \cdot 4$ & $189 \cdot 43$ & $44-4$ & 226.27 & $70 \cdot 7$ & $261 \cdot 71$ & $104 \cdot 8$ & 0.00428 \\
\hline $251 \cdot 27$ & 34.4 & $280 \cdot 40$ & $41 \cdot 4$ & $345 \cdot 44$ & $71 \cdot 5$ & 404.96 & $125 \cdot 3$ & 0.00316 \\
\hline
\end{tabular}

It will be seen that these isometries have much of their general features in common with those of pure carbonic acid and ether* under conditions far apart from their critical points. Several obvious remarks which arise from such a comparison are left to the reader.

The following table, which contains the average values of $(\partial p / \partial t)_{v}$, was constructed from the preceding :-

$$
\left(\frac{\partial p}{\partial t}\right)_{v}
$$

\begin{tabular}{|c|c|c|c|}
\hline$v$. & $0^{\circ}-10^{\circ}$. & $10^{\circ}-30^{\circ}$. & $30^{\circ}-50^{\circ}$. \\
\hline 0.02000 & 0.228 & $0 \cdot 225$ & $0 \cdot 222$ \\
0.01636 & 0.291 & 0.286 & 0.281 \\
0.01300 & 0.388 & 0.381 & 0.373 \\
0.01000 & 0.547 & 0.535 & 0.522 \\
0.00768 & 0.789 & $0 \cdot 765$ & $0 \cdot 744$ \\
0.00578 & $1 \cdot 190$ & $1 \cdot 155$ & $1 \cdot 116$ \\
0.00428 & 1.904 & $1 \cdot 842$ & 1.772 \\
0.00316 & 3.213 & $3 \cdot 102$ & $2 \cdot 976$ \\
\hline
\end{tabular}

In the abstract already referred to, Andrews gives a few measurements relating to a mixture of about 11 volumes of carbonic acid and 1 volume of air, and remarks that "the

* W. Ramsay and S. Young, Phil. Mag. 1887, vol. xxiii.

Phil. Mag. S. 5. Vol. 36. No. 222. Nov. 1893. $2 \mathrm{H}$ 
values of $\alpha\left(\equiv\left(\frac{\partial p}{\partial t}\right)_{v}\right)$, deduced for the same range of temperature from the elastic forces at different pressures, are directly proportional to one another." This remark applies also to the present case. Graphically it means that the isometrics are very nearly straight, and also from equation (1) we have

$$
\left(\frac{\partial p}{\partial t}\right)_{v}=\frac{\mathrm{R}}{v-\alpha}+\frac{\mathrm{K}}{\mathrm{T}^{2}(v+\beta)^{2}}
$$

so that when $\mathrm{T}$ is sufficiently great the first term on the righthand side supersedes the second, and consequently

$$
\frac{\left(\frac{\partial p}{\partial t}\right)_{v^{\prime}}}{\left(\frac{\partial p}{\partial t}\right)_{v^{\prime \prime}}}=\frac{v^{\prime \prime}-\alpha}{v^{\prime}-\alpha}=\text { const. }
$$

Thus the above relation appears less remarkable than at first sight.

Fig. 4.

Isopiestics.

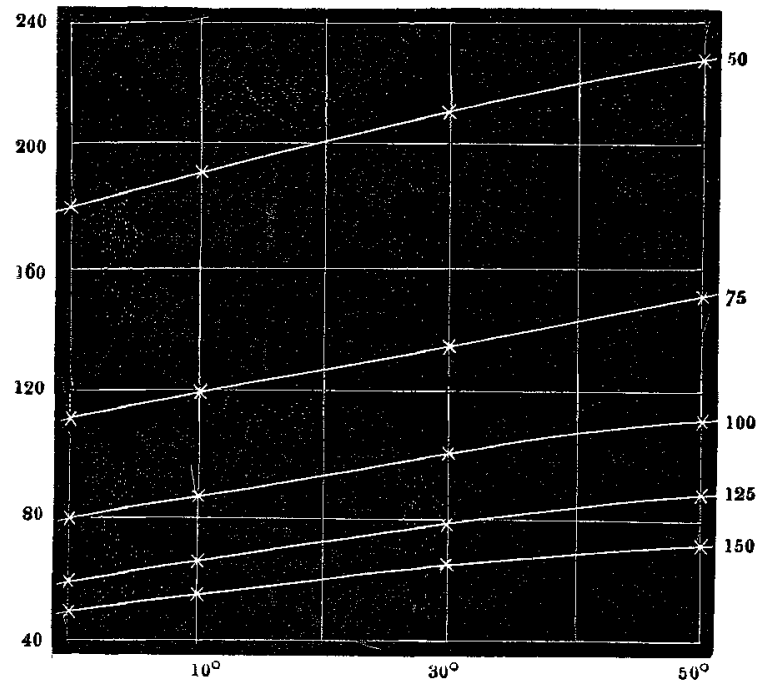

The following Tables give a few numbers relating to isopiestics (see fig. 4). 


\begin{tabular}{|c|c|c|c|c|c|c|c|c|}
\hline \multicolumn{2}{|c|}{$0^{\circ}$. } & \multicolumn{2}{|c|}{$10^{\circ}$. } & \multicolumn{2}{|c|}{$30^{\circ}$. } & \multicolumn{2}{|c|}{$50^{\circ}$. } & \multirow{2}{*}{$p$} \\
\hline Mixture & $\begin{array}{l}\text { Pure } \\
\text { Gas. }\end{array}$ & Mixture. & $\begin{array}{l}\text { Pure } \\
\text { Gas. }\end{array}$ & Mixture. & $\begin{array}{l}\text { Pure. } \\
\text { Gas. }\end{array}$ & Mixture. & $\begin{array}{l}\text { Pure } \\
\text { Gas. }\end{array}$ & \\
\hline 0.01793 & $0.02100 \mid$ & 0.01896 & 002290 & 0.02090 & 0.01550 & 0.02273 & 0.01840 & ${ }_{50}^{\text {atm. }}$ \\
\hline 0.01125 & 0.02047 & 0.01205 & 0.02173 & 0.01352 & 0.00292 & 001487 & 0.00996 & 75 \\
\hline 0.00793 & 0.02020 & 0.00864 & 0.02130 & 0.00988 & 0.00255 & 0.01099 & $0.0049 \mathrm{i}$ & 100 \\
\hline 0.00604 & $\mid 0.01992$ & $0 \cdot 00666$ & 0.02096 & 0.00775 & 0.00240 & 0.00869 & 0.00316 & 125 \\
\hline 0.00489 & $0^{\circ} 01966$ & 0.00542 & $0 \cdot 02060$ & 0.00937 & 0.00230 & 0.00720 & 0.00279 & 150 \\
\hline
\end{tabular}

\section{$\left(\frac{\partial v}{\partial t}\right)_{p}$}

\begin{tabular}{|c|c|c|c|}
\hline$p$. & $0^{\circ}-10^{\circ}$. & $10^{\circ}-30^{\circ}$. & $30^{\circ}-50^{\circ}$. \\
\hline atm. & 0.000103 & 0.000097 & 0.000091 \\
75 & 0.000080 & 0.000074 & 0.000067 \\
100 & 0.000071 & 0.000062 & 0.000055 \\
125 & 0.000062 & 0.000056 & 0.000047 \\
150 & 0.000052 & 0.000047 & 0.000041 \\
\hline
\end{tabular}

\section{SUNDRy Remarks.}

Could we, as is often done, make nse of equation (1) far beyond the region of direct measurements, the critical point would be given by the following equation:-

$$
\mathrm{T}^{2}=\frac{8}{27} \cdot \frac{\mathrm{K}}{(\alpha+\beta) \cdot \mathrm{R}},
$$

which gives in the present case $\mathrm{T}=222^{\circ} .55$; accordingly the critical point of the mixture under consideration would be $-50^{\circ} .45$. As already mentioned, Andrews found it to be below $-20^{\mathrm{C}}$.

One effect of an admixture of carbonic acid with a gas like nitrogen is that isothermals of the former are somewhat deformed *. It seems to me likely, but requires to be decided

* One sees this very clearly even from a few measurements of Janssen on nitrous oxide at $25^{\circ} \cdot 15$ and $32^{\circ} \cdot 2$, as well as from Andrews's on carbonic acid at $13^{\circ} \cdot 1$ and $21^{\circ} 5$. 
experimentally, that as the admixture becomes greater in proportion, the slope of isothermals between the gas- and liquid-lines against the line of constant pressure will become greater.

Graphically speaking, those intermediate portions of isothermals in case of an absolutely pure gas shift parallel to themselves as the temperature becomes higher, and, preserving their straight form till the critical point is neared, at last vanish there, to make up the continuity of curvature of those portions which lie on both sides of them. For a mixture, the slope of those intermediate portions seems to become greater as the temperature becomes higher, and thus that continuity to be smoothly made up. In such a discussion one will get much help from experiments already referred to as made by Cailletet and, if any, others.

The curious phenomenon that a gaseous mixture presents an optically homogeneous appearance once for a while within the region bounded by the gas- and liquid-lines, attracted much attention from physicists. Jamin ascribes it to the mutual approach to equality of densities of the mixed gases due to high pressures ; while Duhem* tries to deduce the existence of, and to explain graphically, that homogeneous appearance from his mathematical equations of thermodynamic potentials. Jamin's explanation is physical to a certain extent, yet seems to be somewhat deficient, while Duhem's may not be deficient, yet seems to be not quite physical. At the same time, Andrews was undoubtedly the first to observe the phenomenon, and his descriptions and experiments seem to contain the right means towards understanding the true physical processes by which the phenomenon under consideration is occasioned. Dewar's experiments on the critical points of mixed vapours seem to direct us for an explanation towards the same point as Andrews's; so also do the experiments of Cailletet.

The course of isothermals of a gaseous mixture much below the critical point may probably not be much different from the usual course. Andrews observed near the point of complete condensation how a little mass of nitrogen diffuses into the condensed liquid. A little increase of volume due to that diffusion must be of the same nature, as was pointed out by Angström $†$. In the meantime, evaporation, a special type of diffusion, of the condensed liquid into the little atmosphere of nitrogen must have been going on under the special conditions of temperature and pressure.

These interdiffusions of one part into another of the hetero-

* Journ. de Phys. vii. 1888.

$\dagger$ Wied. Ann. Bd. xvii. 1882. 
geneous mass must have presented themselves in a very striking manner at higher temperatures and under stronger pressures, and at a certain stage resulted in the production of the above optically homogeneous appearance.

It is therefore very likely, as Jamin remarked, that we have under those circumstances of temperature and pressure nothing that is different in kind from what we see under ordinary circumstances.

Dewar's experiments, especially those on liquefaction of carbonic acid in presence of other liquids, are interesting from our point of view, and we cannot but think of some such process as interdiffusion being in action through all cases, not excepting the case of pure gases.

XLVI. On the Theory of Pyro-electrieity and Piezoelectricity of Crystals. By Lord Kelvis*.

$\S 1 . T$ THE doctrine of bodily electro-polarization masked by an induced superficial electrification, which I gave thirty years ago in Nichol's Cyclopacdia $\dagger$, wanted a physical explanation of the assumed molecular polarization to render it a satisfying physical theory of pyro-electricity; and it was essentially defective, as has been remarked by Röntgen $\ddagger$ and by Voigt $\S$, in that it contained no suggestion towards explaining the multiple electric polarities irregularly produced by irregular changes of temperature in boracite, in quartz, and in tourmaline itself; which had perplexed many naturalists and experimenters. A short but very important paper by MM. Jacques and Pierre Curie in the Comptes Rendus for Feb. 14, 1881, supplies that want in a manner which suggests what seems to me the true matter-of-fact electro-chemical theory of a crystalline molecule, and at the same time makes easy the extension of my slight primitive doctrine, to remedy its defect in respect to multipolarity, and to render it available for explaining not only the old-known pyro-electricity of crystals, but also the piezo-electricity discovered by the brothers Curie $\|$ themselves. The element of zinc and copper soldered together and surrounded only by air, which they suggest, represents perfectly a true electro-

* Communicated by the Author.

+ Reprinted in 'Collected Mathematical and Physical Papers' (Sir W. Thomson), vol. i. p. 315.

i Wiedemann's Annalen, 1883, xviii. p. 213.

$\$$ "Allgemeine Theorie der Pjëzo- und Pyro-electrischen Erscheinungen an Krystallen," p. 6 ; separate publication from vol. xxxvi. of Abhand. König. Ges. Wiss. Göttingen, 1890.

If Comptes Rendus, Aug. 2 and Aug. 16, 1880. 